\title{
Energy diffusion in strongly driven quantum chaotic systems
}

\author{
P.V. Elyutin* \\ Department of Physics, Moscow State University, Moscow 119992, Russia
}

(Dated: November 21, 2018)

\begin{abstract}
The energy evolution of a quantum chaotic system under the perturbation that harmonically depends on time is studied for the case of large perturbation, in which the rate of transition calculated from the Fermi golden rule exceeds the frequency of perturbation. It is shown that the energy evolution retains its diffusive character, with the diffusion coefficient that is asymptotically proportional to the magnitude of perturbation and to the square root of the density of states. The results are supported by numerical calculation. They imply the absence of the quantum-classical correspondence for the energy diffusion and the energy absorption in the classical limit $\hbar \rightarrow 0$.
\end{abstract}

PACS numbers 05.45.-a

*Electronic address: pve@shg.phys.msu.su 


\section{INTRODUCTION}

The problem of susceptibility of chaotic systems to perturbations has attracted much attention in the last decade [1 - 9]. This problem is fundamental, since it includes the determination of the response of a material system to an imposed external electromagnetic field, the setup that is typical for many experiments. Due to the sensitivity of classical phase trajectories or quantum energy spectra and stationary wavefunctions of chaotic systems to small changes of their parameters, the problem is challengingly difficult. A consistent and noncontroversial picture covering (albeit qualitatively) all the essential cases of the response hasn't been yet drawn at present. From the point of view of general theory, the problem is related to the applicability of the concept of quantum-classical correspondence to chaotic systems, that is a long-standing question in its own right [10, 11].

We shall study a one-particle system with the Hamiltonian of the form $\hat{H}=\hat{H}_{0}-$ $F \hat{x} \cos \omega_{0} t$, where $\hat{H}_{0}(\hat{\mathbf{p}}, \hat{\mathbf{r}})$ is the Hamiltonian of the unperturbed system; $\hat{\mathbf{p}}$ and $\hat{\mathbf{r}}$ are the operators of Cartesian components of the momentum and of the position of the particle. The classical system with the Hamiltonian function $H_{0}(\mathbf{p}, \mathbf{r})$ will be assumed to be strongly chaotic, that is, nearly ergodic on the energy surfaces in a wide range of the energy values, system with $d \geq 2$ degrees of freedom. In the perturbation operator $\hat{V}(t)=-F \hat{x} \cos \omega_{0} t$ the active variable $\hat{x}$ is one of the Cartesian coordinates of the particle, coupled to the external homogeneous force field. The amplitude $F$ in the following will be referred to as field. In the following we shall deal with the quasiclassical case, when the Planck constant is small in comparison of the action scale of the system $H_{0}$.

Under the influence of the perturbation the energy value $E(t)=H_{0}(t)$ varies in a quasirandom way. These variations frequently can be described as a process of the energy diffusion [12, 13], when for the ensemble with the microcanonical initial energy distribution $H_{0}(0)=E$ the dispersion of the energy increases linearly with time, $\left\langle\Delta E^{2}(t)\right\rangle=2 D t$, where $D\left(E, F, \omega_{0}\right)$ is the energy diffusion coefficient.

If the external field $F$ is sufficiently small in comparison with the appropriately averaged values of the forces acting on a particle in the unperturbed system, then in the classical model the energy diffusion coefficient $D$ can be expressed through the characteristics of the unperturbed chaotic motion of the active coordinate, namely

$$
D=\frac{\pi}{2} \omega_{0}^{2} F^{2} S_{x}\left(E, \omega_{0}\right)
$$

where $S_{x}\left(E, \omega_{0}\right)$ is the power spectrum of the active coordinate (the Fourier transform of its autocorrelation function) for the motion on the surface with the constant energy value $E$ [9]. 
The same expression (1) in the case of weak perturbation can be obtained in the classical limit from the quantum model. The evolution of the quantum system can be treated as a sequence of one-photon transitions between stationary states of the unperturbed system $|n\rangle \rightarrow|k\rangle$, accompanied with the absorption or emission of the quanta $\hbar \omega_{0}$. For small $\hbar$ the energy spectrum of $\hat{H}_{0}$ is quasicontinuous, thence the rates of transition are given by the Fermi golden rule (FGR)

$$
\dot{W}_{F}=\frac{\pi}{2 \hbar} F^{2}\left|x_{n k}\right|^{2} \rho\left(E_{k}\right),
$$

where $x_{n k}$ is the matrix element of the active coordinate, and $\rho\left(E_{k}\right)$ is the density of states near the final state of the transition. Although the matrix elements $x_{n k}$ in quantum chaotic systems fluctuate wildly with the variation of $k[10,11]$, the averaged squared quantity $\overline{\left|x_{n k}\right|^{2}}$ in the limit $\hbar \rightarrow 0$ is smooth; it is proportional to the power spectrum $S_{x}\left(E, \omega_{0}\right)$ of the coordinate [14, 15],

$$
\overline{\left|x_{n k}\right|^{2}} \approx \frac{S_{x}\left(E, \omega_{0}\right)}{\hbar \rho(E)} .
$$

From Eqs. (2) and (3) we have for the transition rate

$$
\dot{W}_{F}=\frac{\pi}{2 \hbar^{2}} F^{2} S_{x}(E, \omega)
$$

Then for the energy dispersion for small $t$ we have $\left\langle\Delta E^{2}\right\rangle=2\left(\hbar \omega_{0}\right)^{2} \dot{W}_{F} t$, that returns us to the Eq.(1) for the energy diffusion coefficient. It can be shown that the same expression for $D$ holds also for large $t$ [9]. The energy absorption in chaotic systems comes as an epiphenomenon of the energy diffusion [4]. With the account of the dependence on the energy of the power spectrum $S_{x}(E, \omega)$ and the density of states $\rho(E)$ the diffusion becomes biased, and the energy absorption rate $Q$ is given by the formula [2, 4]

$$
Q=\frac{1}{\rho} \frac{d}{d E}(\rho D)
$$

Although for weak fields $D$ does not depend on the Planck constant $\hbar$, the condition of the applicability of Eq. (2) does. The FGR is, after all, only a formula of the first order perturbation theory. It is based on the assumption that the transition process has a resonant character - that the width $\Delta$ of the energy distribution of states populated from the original one, given by the Weisskopf - Wigner formula $\Delta=\hbar \dot{W}$ [16], is small in comparison with the quanta energy $\hbar \omega_{0}$. From Eq. (4) it is evident that in the classical limit $\hbar \rightarrow 0$ this condition will be violated. In the following we shall use the border value of the field $F_{b}$, defined by the condition $\dot{W}_{F}\left(F_{b}\right)=\omega_{0}$, and refer to the domain $F \geq F_{b}$ as the range of the strong field. 
By analogy with other models, for strong fields one can expect a slow-down of the growth of the energy diffusion coefficient $D$ and of the energy absorption rate $Q$. For example, for a two-level system with relaxation the quadratic dependence the absorption rate $Q \propto F^{2}$ for small field turns into a field-independent value $Q_{0}$ for strong one. The border is determined by the condition $\Omega^{2} / \Gamma_{1} \Gamma_{2} \sim 1$, where $\Omega$ is the Rabi frequency and $\Gamma_{1} \Gamma_{2}$ are longitudinal and transversal relaxation rates correspondingly [17]. The rate of transitions from discrete to continuous energy spectrum (that are basically covariant with the energy absorption rate $Q$ ), studied in the context of the theory of photoionization, for sufficiently strong fields can even decrease with the increase of $F$ - the effect that is known as atom stabilization by the strong field [18].

For our model we can describe the slow-down by a simple estimate. Assuming the exponential decay of the initially populated state and using the Weisskopf - Wigner formula for the energy distribution of the final states [16], we can rewrite the expression for the rate of the transitions (with the absorption of quanta) in the form

$$
\dot{W}=\frac{\pi}{2} \int \frac{V^{2}(\omega)}{\hbar}\left[\frac{1}{\pi} \cdot \frac{\dot{W}}{\left(\omega-\omega_{0}\right)^{2}+\dot{W}^{2}}\right] \rho(\omega) d \omega,
$$

where $V(\omega)$ is a matrix element of perturbation taken as a function of the transition frequency and $\omega=\left(E_{k}-E_{n}\right) / \hbar$. In the weak field the expression in square brackets turns into the $\delta$ - function, and Eq. (6) returns the usual FGR. However, if we dare to use Eq. (6) as an equation that is valid for any magnitude of the perturbation, then in the case of strong fields we will have

$$
\dot{W}^{2}=\frac{1}{2} \int \frac{V^{2}(\omega)}{\hbar} \rho(\omega) d \omega .
$$

The rate of transitions $\dot{W}$, and, consequently, the energy diffusion coefficient $D$ and the energy absorption rate $Q$, become proportional to the magnitude of perturbation $F$ and to the square root of the density of states. This derivation of Eq. (7) is too risky - but in the following sections this conclusion will be put on a more solid footing.

The slow-down of the energy diffusion in quantum chaotic systems in strong harmonic fields has been first demonstrated by Cohen and Kottos [5]. However, their analytical estimates and data of numerical experiments are in quantitative disagreement with the results of the present paper.

Lastly, it must be noted that the strong field regime is easily attainable to experiments. For example, for the excitation of multiatomic molecules with the infrared laser radiation the border field corresponds to the intensity value $I \sim 10^{9} \mathrm{~W} \mathrm{~cm}^{-2}$, that has been reached in experiments long time ago [19]. 


\section{THEORY}

For the system with the Hamiltonian $\hat{H}=\hat{H}_{0}+\hat{V} \cos \omega_{0} t$ we take the wave function in the form of the expansion in the basis of stationary $\left\{\varphi_{m}\right\}$ states of $\hat{H}_{0}$,

$$
\Psi(t)=\sum_{m} a_{m} \varphi_{m}(\mathbf{r}) e^{-i \omega_{m} t}
$$

Then for the amplitudes $\left\{a_{m}(t)\right\}$ we obtain the system of equations

$$
i \frac{d a_{k}}{d t}=\sum_{k} \Omega_{k m} \cos \omega_{0} t e^{i \omega_{k m} t} a_{m}
$$

where the quantities $\Omega_{k n}=\hbar^{-1} F x_{k n}$ are the Rabi frequencies of transitions. We shall use the initial conditions $a_{m}(0)=\delta_{m n}$ : at the initial moment only one of stationary states, $\varphi_{n}$, is populated. Following [14, 15], we shall assume that $x_{n k}$ are independent random Gaussian variables with zero mean value and the dispersion, given by Eq. (3). The system of equations (9) will be treated as a member of the corresponding statistical ensemble.

We are going to concentrate on the process of the energy diffusion. Then in the zeroth approximation we can restrict ourselves by consideration of the evolution of probability density in a narrow energy range around the initial state and use the power spectrum and the density of states values at this energy: $S_{x}(\omega) \equiv S_{x}\left(E_{n}, \omega\right)$ and $\rho \equiv \rho\left(E_{n}\right)$. For the calculation of the absorption coefficient the global dependence on the energy must be restituted.

The power spectrum $S_{x}(\omega)$ has the symmetry property $S_{x}(-\omega)=S_{x}(\omega)$. The dependence $S_{x}(\omega)$ in the domain $\omega>0$ in typical strongly chaotic systems, such as nonlinear oscillators [20] and billiards [21, 22], has the form of an asymmetric peak. We shall define the peak value of the Rabi frequency simply as $\Omega$, the frequency of the maximum as $\tilde{\omega}$ and the characteristic width of the peak as $\Gamma$. Typically the ratio $\tilde{\omega} / \Gamma$ is about few units.

Right after the switching on the perturbation all amplitudes (but that of the initially populated state) grows in absolute value linearly in time. At this ballistic stage the energy dispersion grows quadratically in time:

$$
\left\langle\Delta E^{2}\right\rangle \approx K_{1} \hbar^{3} \tilde{\omega}^{2} \Omega^{2} \Gamma \rho t^{2}
$$

where $K_{1}$ is a numerical constant. This stage is limited by the depletion of the initial population and lasts until the depletion time $t_{d} \sim \Omega^{-1}(\hbar \Gamma \rho)^{-1 / 2}$. At this time considerably populated levels are spread over the energy range $\Delta E \sim \hbar \tilde{\omega}$ that contains many levels (since $\rho \propto \hbar^{-d}$ with $d \geq 2$ ). We shall expect that at the next stage the ensemble averaged density of probability is a smooth function with a characteristic scale $\Delta E>>\hbar \tilde{\omega}$. 
It is convenient to write indices in Eq. (9) as arguments of functions. We shall use the frequency distance from the initial level as a basic independent variable $\omega, a_{k}$ will be denoted as $a(\epsilon)$, where $\varepsilon=\left(E_{k}-E_{n}\right) / \hbar$. Dummy variables $\eta$ and $\eta^{\prime}$ will have the same meaning. By formal integration of Eq. (9) and subsequent recurrent substitution we obtain the equation for the rate of change of the local probability density $w(\varepsilon)=|a(\varepsilon)|^{2}$ :

$$
\begin{aligned}
& \frac{d w(\varepsilon)}{d t}=\sum_{\eta, \eta^{\prime}} \Omega(\varepsilon, \eta) e^{i(\varepsilon-\eta) t} \cos \omega_{0} t a(\eta, t) \times \\
\times & \int d t^{\prime} \Omega\left(\varepsilon, \eta^{\prime}\right) e^{-i\left(\varepsilon-\eta^{\prime}\right) t} \cos \omega_{0} t a^{*}\left(\eta^{\prime}, t\right)+\text { c.c. }
\end{aligned}
$$

Summation in this formula goes over discrete values of $\eta$ and $\eta^{\prime}$, and this equation is still exact.

Now we assume, that the amplitudes $a(\eta, t)$ are random processes, that are not correlated for different states: $\left\langle a(\eta, t) a^{*}\left(\eta^{\prime}, t^{\prime}\right)\right\rangle \propto \delta_{\eta \eta^{\prime}}$. Then for the averaged probability density we can retain in the Eq. (11) only diagonal terms:

$$
\begin{array}{r}
\frac{d\langle w(\varepsilon)\rangle}{d t}=\sum_{\eta}\left\langle\Omega^{2}(\varepsilon, \eta)\right\rangle \cos \omega_{0} t \times \\
\times \int^{t} d t^{\prime} e^{i(\varepsilon-\eta)\left(t-t^{\prime}\right)} \cos \omega_{0} t^{\prime}\left\langle a(\eta, t) a^{*}\left(\eta, t^{\prime}\right)\right\rangle+\text { c.c. }
\end{array}
$$

With the assumption that the averaged $\langle w(\varepsilon, t)\rangle$ is a smooth function of $\varepsilon$, and slowly varying function of $t$, we may cast the product of amplitudes in the form

$$
\left\langle a(\eta, t) a^{*}\left(\eta, t^{\prime}\right)\right\rangle+\text { c.c. }=2\langle w(\eta, t)\rangle B\left(t-t^{\prime}\right),
$$

where $B(\tau)$ is the normalized $(B(0)=1)$ autocorrelation function of the probability amplitudes.

By replacing the averaged square Rabi frequency by its value from Eq. (3) (that depends only on the difference $\varepsilon-\eta$ ), substituting the summation over the states by the integration weighted with the density of states, and averaging over the time intervals that are much larger than the field period, we come to the equation

$$
\frac{d w(\varepsilon)}{d t}=\int d \eta \Omega^{2}(\varepsilon-\eta) \rho(\eta) \int_{0}^{\infty} d \tau \cos (\varepsilon-\eta) \tau \cos \omega_{0} \tau B(\tau) w(\eta)
$$

From now we drop the angular brackets and deal only with ensemble averaged quantities. If the rate of variations of $w(\eta, t)$ is small in comparison with the decay of correlations of 
amplitudes given by $B(\tau)$, then we can treat Eq. (13) as summation over the probability flow that comes from the different parts of the frequency range with the constant rate,

$$
\dot{W}(\eta \rightarrow \varepsilon)=\Omega^{2}(\varepsilon-\eta) \int_{0}^{\infty} d \tau \cos (\varepsilon-\eta) \tau \cos \omega_{0} \tau B(\tau)
$$

This approximate expression to some extent replaces the Fermi golden rule for strong perturbations.

To construct the kinetic equation one must take into account both incoming and outgoing flows of probability. By taking into account the total probability flow, expanding $w(\varepsilon)$ in the Taylor series, we obtain a diffusion equation with the probability diffusion coefficient in the energy scale

$$
D \approx \int_{-\infty}^{\infty} d \eta \hbar^{3} \eta^{2} \Omega^{2}(\eta) \rho J\left(\eta, \omega_{0}\right)
$$

where

$$
J\left(\eta, \omega_{0}\right)=\int_{0}^{\infty} d \tau \cos \eta \tau \cos \omega_{0} \tau B(\tau) .
$$

Now the problem reduces to the calculation of the integral $J\left(\eta, \omega_{0}\right)$. For large enough times the average probability density, that is governed by the diffusion equation, varies slowly, and we can treat the system Eq. (9) as a set of equations in which all $a_{m}(t)$ are non-correlated random processes with the same statistical properties. Then by averaging the equation for the squared time derivative of an amplitude, we obtain the expression for the mean squared frequency of these processes, that also gives the estimate for the square of the correlation decay rate $\gamma$ :

$$
\left\langle\omega^{2}\right\rangle=\frac{1}{2} \int \Omega^{2}(\eta) \hbar \rho d \eta \equiv \gamma^{2}
$$

From Eq. (18) for the decay correlation rate we have the estimate

$$
\gamma \approx K_{2} \Omega \sqrt{\Gamma \hbar \rho}
$$

where $K_{2}$ is a numerical constant In the domain of strong field the autocorrelation function is the fastest component under the integration sign in Eq. (17). Then we have $J \sim \gamma^{-1} \approx$ $\left(K_{2} \Omega \sqrt{\Gamma \hbar \rho}\right)^{-1}$. By the substitution of this expression into Eq. (16) we obtain the estimate of the energy diffusion coefficient:

$$
D \approx K_{3} \hbar^{2} \tilde{\omega}^{2} \Omega \sqrt{\Gamma \hbar \rho}
$$

where $K_{3}$ is a numerical constant; this expression agrees with the dependence that was derived in Sec. 1. One must recall that this expression is valid only for the nearly resonant 
perturbation frequency $\left(\left|\omega_{0}-\tilde{\omega}\right| \leq \Gamma\right)$. We do not enter here into the studies of the dependence of $D$ on the perturbation frequency $\omega_{0}$ in the wider domain, postponing it for the further studies.

\section{NUMERICAL EXPERIMENT}

To check the analytical results of the preceding section, the system of equations (9) has been integrated numerically. The number of equations varied from $N=300$ to $N=$ 1200 with the purpose to suppress the influence of the border. The envelope of the Rabi frequencies has been taken in the double lorentzian form

$$
\Omega_{m, n}=\Omega\left[\frac{\Gamma^{2}}{\left(\omega_{m}-\omega_{n}+\tilde{\omega}\right)^{2}+\Gamma^{2}}+\frac{\Gamma^{2}}{\left(\omega_{m}-\omega_{n}+\tilde{\omega}\right)^{2}+\Gamma^{2}}\right] .
$$

All calculations have been carried for the "resonant" perturbation frequency $\omega_{0}=\tilde{\omega}$ and for the peak width $\Gamma=0.3 \tilde{\omega}$.

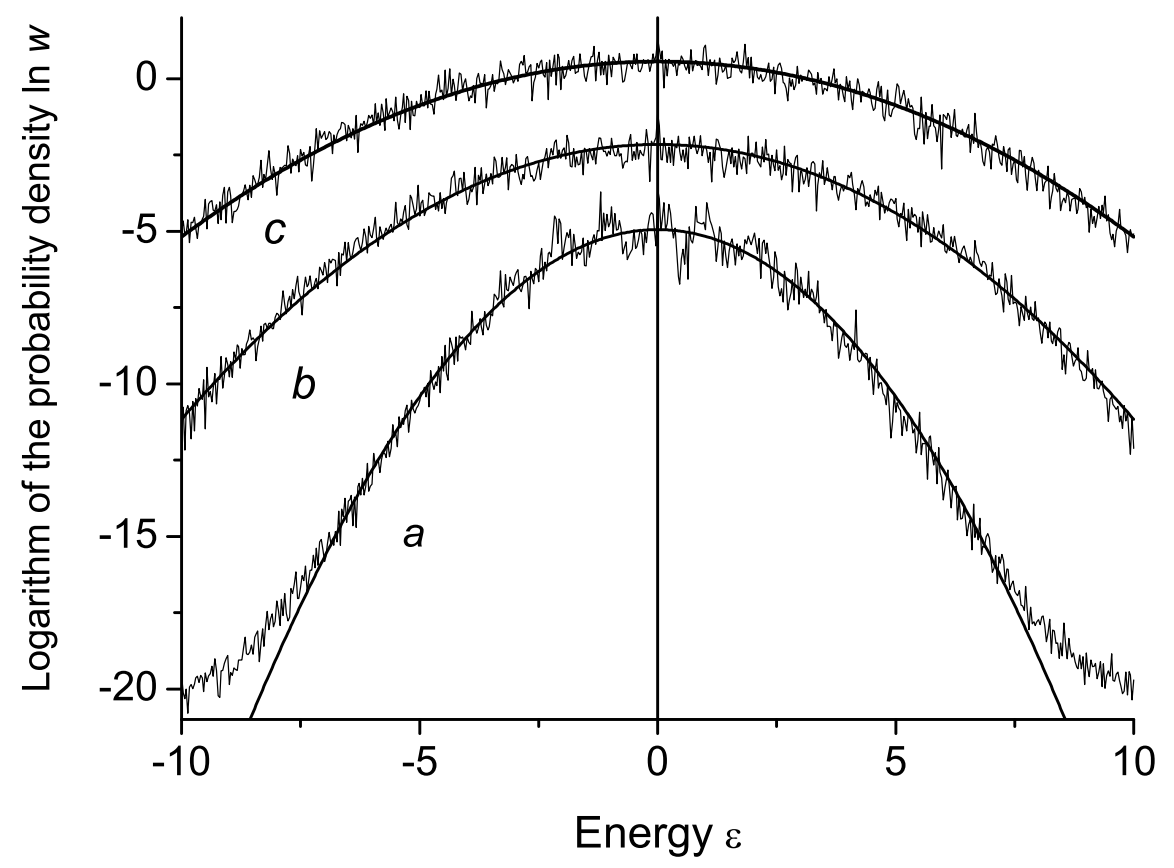

FIG. 1: The dependence of the logarithm of the probability density on the dimensionless frequency $\varepsilon^{\prime}=\varepsilon / \tilde{\omega}$ for the time values $t=5 \tilde{\omega}^{-1}$ (a), $t=10 \tilde{\omega}^{-1}(\mathrm{~b})$, and $t=15 \tilde{\omega}^{-1}$ (c). The grassy lines values of $\ln w\left(\varepsilon^{\prime}\right)$, averaged over 10 different sets of matrix elements, solid lines - fitted parabolas. To avoid the overlap of graphs, the plots for cases (b) and (c) are shifted upwards by 3 and 6 units respectively. 
Fig. 1 shows the distribution of probability as a function of dimensionless frequency $\varepsilon^{\prime}=\varepsilon / \tilde{\omega}$ for different moments of time. It is clearly seen that even for relatively small time $t=5 \tilde{\omega}^{-1}=2.2 t_{d}$ the distribution has very accurate Gaussian form, with deviations noticeable only for $\left|\varepsilon^{\prime}\right| \geq 7.5$. Thus we have quantitative support for our conclusion about the diffusive character of the energy evolution.

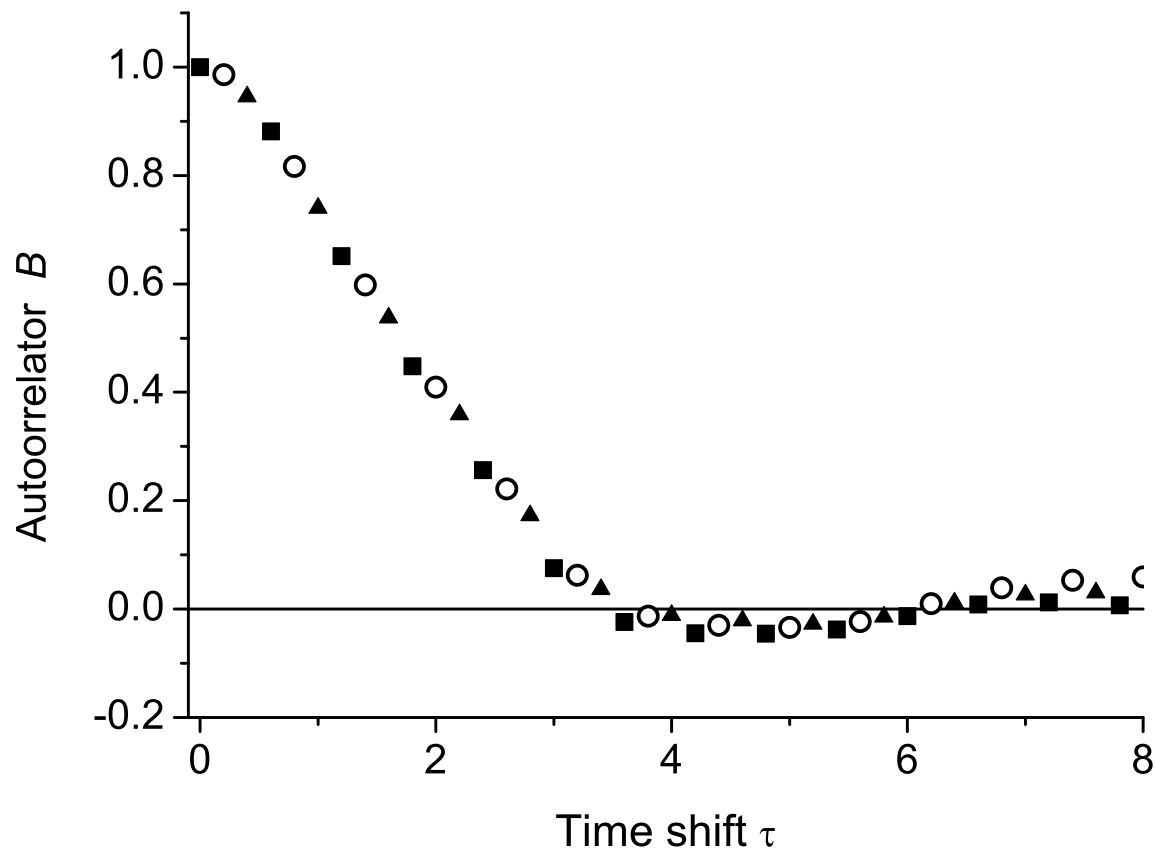

FIG. 2: Dependence of the autocorrelation function of the probability amplitudes on the dimensionless time shift $\tau^{\prime}=\tau \tilde{\omega}$ for three different sets of parameters with $\tilde{\omega} \hbar \rho=30$ (black squares), $\tilde{\omega} \hbar \rho=60$ (open circles) and $\tilde{\omega} \hbar \rho=120$ (black triangles) and the same value of $\Omega \sqrt{\Gamma \hbar \rho}=0.618 \tilde{\omega}$. The statistical errors are about the size of the data symbols.

Fig 2 depicts the form of the normalized autocorrelation function of the probability amplitudes. Values $B(\tau)$ have been calculated numerically for three sets of parameters with different values of $\rho$ but the same value of the product $\Omega \sqrt{\hbar \Gamma \rho}$. It is clearly seen that $B(\tau)$ for these sets are nearly identical, as expected. The decay rate $\gamma$ taken from the equation $B\left(\gamma^{-1}\right)=\exp (-1)$ is $\gamma=0.77 \Omega \sqrt{\hbar \Gamma \rho}$, that supports the estimate Eq. (19). 


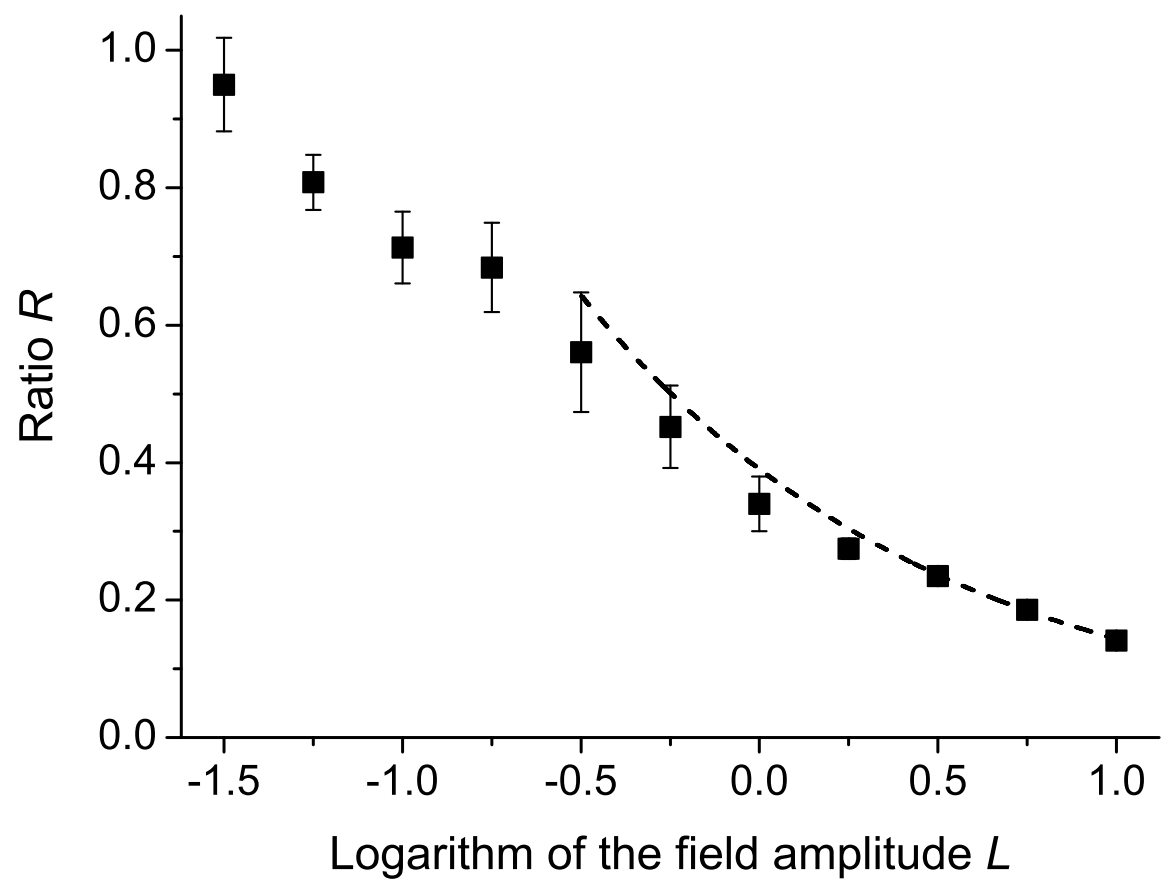

FIG. 3: The dependence of the ratio $R=D / 2 \dot{W}_{F}$ of the energy diffusion constant $D$ to the doubled Fermi transition rate $\dot{W}_{F}=(\pi / 2) \Omega^{2} \hbar \rho$ on the logarithm of the ratio of the Rabi frequency to its border value $L=\Omega / \Omega_{b}, \Omega_{b}=(2 \tilde{\omega} / \pi \hbar \rho)^{1 / 2}$. The dashed line represents the curve $R=A \exp (-L)$ that corresponds to the theoretical dependence Eq. (20); it is fitted to the last three points.

Figure 3 represents the dependence of the ratio $R=D / 2 \dot{W}_{F}$ of the energy diffusion constant $D$ to the doubled Fermi transition rate $\dot{W}_{F}=(\pi / 2) \Omega^{2} \hbar \rho$ on the logarithm of the ratio of the Rabi frequency to its border value $L=\Omega / \Omega_{b}, \Omega_{b}=(2 \tilde{\omega} / \pi \hbar \rho)^{1 / 2}$. It is seen that for the weak field this ratio comes close to the asymptotic limit (unity), decreases in the vicinity of the border and decays as $F^{-1}$ for sufficiently strong fields. The agreement with the theoretical estimates is quite convincing.

\section{CONCLUSION}

From the comparison of the numerical data with the theoretical estimates we can conclude that the approach of the Sec. 2 gives reasonably accurate description of the energy evolution process in strong fields, in spite of numerous simplifying approximations used in the calculation.

To improve the accuracy and, firstly, to derive from the first principles the equation for 
the correlation function $B(\tau)$, the model of the random process must be improved. We used the model of the stationary process, whereas from Eq. (9) one can conclude that the model of periodic random process would be more appropriate.

The main conclusion from the results of our calculation is a qualitative one: by substituting Eq. (3) in Eq. (16), we obtain $D \approx K_{3} \hbar \tilde{\omega}^{2} F\left[S_{x}\left(\omega_{0}\right) \Gamma\right]^{1 / 2}$. This quantity in the classical limit $\hbar \rightarrow 0$ vanishes along with the energy absorption rate $Q$ (see Eq. (5)). That means the violation of the quantum-classical correspondence for the absorption - and, more generally, for the linear susceptibility of chaotic systems to harmonic external fields.

\section{Acknowledgements}

The author acknowledges the support by the "Russian Scientific Schools" program (grant \# NSh - 1909.2003.2).

\section{References}

[1] N. Brenner and Sh. Fishman 1996 Phys. Rev. Lett. 773763

[2] P.V. Elyutin and J. Shan 1996 Phys. Rev. Lett. 775043

[3] Sh. Mukamel, V. Khidekel, and V. Chernyak 1996 Phys. Rev. E 53 R1

[4] D. Cohen 1999 Phys. Rev. Lett. 824951

[5] D. Cohen and T. Kottos 2000 Phys. Rev. Lett. 854839

[6] A. Iomin 2000 Phys. Rev. E 62442

[7] K. Morawetz 2000 Phys. Rev. E 612555

[8] Ch.H. Reick 2002 Phys. Rev. E 66036103

[9] P.V. Elyutin 2004 Phys. Rev. E 69036207

[10] B. Eckhardt 1988 Phys. Reports 163205

[11] P.V. Elyutin 1988 Uspekhi Fiz. Nauk 155397

[12] S. Linkwitz and H. Grabert 1991 Phys. Rev. B-I 4411888

[13] C. Jarzynski 1993 Phys. Rev. E 484340

[14] M. Feingold and A. Peres 1986 Phys. Rev. A 34591

[15] M. Wilkinson 1987 J. Phys. A: Math. Gen. 202415

[16] V. Weisskopf and E. Wigner 1930 Zs. Phys. 6354 
[17] L. Allen and J.H. Eberly 1975 Optical Resonance and Two-Level Atoms (NY, John Wiley and Sons, 1975)

[18] N.B. Delone and V.P. Krainov 1995 Uspekhi Fiz. Nauk 1651295

[19] V.S. Letokhov and A.A. Makarov 1981 Uspekhi Fiz. Nauk 13445

[20] P.V. Elyutin and V.G. Korolev 1989 Vestn. Mosk. Univ. 3 Fiz. Astron. 3087

[21] T. Prosen and M. Ronbik 1993 J. Phys. A Math. Gen. 26 L319

[22] P.L. Garrido and G. Gallavatti 1994 J. Stat. Phys. 76549 